\title{
State-of-Charge Estimation for Li-ion Batteries: A More Accurate Hybrid Approach
}

Misyris, George; Doukas, Dimitrios I.; Papadopoulos, Theofilos A.; Labridis, Dimitris P.; Agelidis, Vassilios G.

Published in:

IEEE Transactions on Energy Conversion

Link to article, DOI:

10.1109/TEC.2018.2861994

Publication date:

2018

Document Version

Peer reviewed version

Link back to DTU Orbit

Citation (APA):

Misyris, G., Doukas, D. I., Papadopoulos, T. A., Labridis, D. P., \& Agelidis, V. G. (2018). State-of-Charge Estimation for Li-ion Batteries: A More Accurate Hybrid Approach. IEEE Transactions on Energy Conversion, 34(1), 109 - 119. https://doi.org/10.1109/TEC.2018.2861994

\section{General rights}

Copyright and moral rights for the publications made accessible in the public portal are retained by the authors and/or other copyright owners and it is a condition of accessing publications that users recognise and abide by the legal requirements associated with these rights.

- Users may download and print one copy of any publication from the public portal for the purpose of private study or research.

- You may not further distribute the material or use it for any profit-making activity or commercial gain

- You may freely distribute the URL identifying the publication in the public portal 


\title{
State-of-Charge Estimation for Li-ion Batteries: A More Accurate Hybrid Approach
}

\author{
George S. Misyris, Student Member, IEEE, Dimitrios I. Doukas, Member, IEEE, \\ Theofilos A. Papadopoulos, Senior Member, IEEE, Dimitris P. Labridis, Senior Member, IEEE \\ and Vassilios G. Agelidis, Fellow, IEEE
}

\begin{abstract}
Modeling of battery energy storage systems (BESS) used for applications, such as electric vehicles and smart grids, emerged as a necessity over the last decade and depends heavily on the accurate estimation of battery states and parameters. Depending on the battery-cell type and operation, a combination of algorithms is used to identify battery parameters and define battery states. This paper deals with robust Li-ion batteries modeling with a specific focus on a hybrid approach for a more accurate state-of-charge (SOC) estimation. The analysis presents a detailed description of the state-of-the-art stand-alone SOC estimation methods and focuses on a hybrid SOC estimation technique to improve accuracy under varying conditions. Emphasis is given on performance improvements of the proposed hybrid approach compared to the conventional methods, whereas a thorough experimental validation is presented to evaluate the accuracy of the proposed method.
\end{abstract}

Index Terms-Battery energy storage systems (BESS), capacity estimation, Coulomb Counting, equivalent circuit model (ECM), model-based methods, parameter identification, state estimation algorithms, state-of-charge (SOC) estimation.

\section{INTRODUCTION}

$\mathbf{L}$ I-ion batteries have been recently deployed in a wide range of energy-storage applications, ranging from energy-type batteries of a few kilowatt-hours in residential systems to multi-megawatt batteries for the provision of grid ancillary services [1]. This trend brought to the fore, a series of requirements in high-energy and high-power applications, which strongly depend on the accurate state-of-charge (SOC) estimation [2]. Accurate SOC estimation contributes to better protected battery packs against over-charging/discharging [3]. Failure to estimate SOC accurately may result in a reduction of the power-output capability and the whole energy management system might be lowered severely, as discussed in [4].

Based on the literature, SOC is defined as the present battery capacity and usually is expressed as a percentage of a reference capacity [5]. The preferred SOC reference can be either the rated capacity of a new battery or more often the current

G. S. Misyris, D. I. Doukas and D. P. Labridis are with the School of Electrical and Computer Engineering, Aristotle University of Thessaloniki, 54124 Thessaloniki, Greece (e-mail: doux@auth.gr).

T. A. Papadopoulos is with the Power Systems Laboratory, Department of Electrical and Computer Engineering, Democritus University of Thrace, 67100 Xanthi, Greece (e-mail: thpapad@ee.duth.gr).

G. S. Misyris and V. G. Agelidis are with the Department of Electrical Engineering, Technical University of Denmark, 2800 Kgs. Lyngby, Denmark (e-mail: vasagel@elektro.dtu.dk).

*Part of this work has been done while G. S. Misyris was with ABB Corporate Research, 72178 Västerås, Sweden.

Manuscript submitted July 30, 2018. maximum capacity of the battery. Several approaches have been proposed regarding SOC estimation of Li-ion batteries. Among them the Coulomb Counting (CC) [3], [6], the socalled open circuit voltage (OCV)-based methods [7], [8] and the model-based methods [9] have mainly drawn the attention of researchers over the last decades.

Model-based methods provide in the long term robust results and are more accurate compared to CC and OCV-based methods [5]. However, they cannot compete the accuracy of the CC method in the short-term or of the OCV-based approaches after a long relaxation period of the battery [10]. To that end, the SOC estimation methods can be categorized in terms of the estimation model/algorithm, advantages, drawbacks, estimation accuracy and computational burden. More recently, in [11] and [12], SOC estimation alternatives have been summarized and classified based on different criteria.

A number of hybrid approaches related to SOC estimation can be found in the literature as well. In [13], particle filter and multi-models data fusion methodologies are combined for a robust real-time SOC estimation tool that outperforms stand-alone model-based techniques. Moreover, in [14], an alternative approach that is based on iterated extended Kalman particle filter (IEKPF) is proposed, achieving SOC estimation results of high accuracy.

The scope of this paper is to provide a holistic modeling approach for Li-ion batteries, especially for online applications. The detailed mathematical formulation for all modeling parts is described, whereas focus is given on the SOC estimation module. Regarding parameter identification and capacity estimation, the Fast Upper-triangular and Diagonal Recursive Least Squares (FUDRLS) with varying forgetting factors [15] and the Approximate Weighted Total Least Squares (AWTLS) algorithms are used [16]. Regarding the SOC estimation, CC, Linear Kalman Filter (LKF) and OCV-based methods are utilized, either stand-alone or combined to hybrid setups.

The contribution of this paper is to propose a new hybrid accurate SOC estimation method by combining different methods presented in the literature. The proposed solution ensures safe battery operation within the acceptable SOC limits and prolong its lifetime. Different estimation techniques advantages are combined towards SOC error minimization, limiting the computational burden and thus making the proposed method suitable for online applications. A detailed mathematical formulation for the SOC estimation module and the other modeling parts is presented. Extensive experimental validation, based on different setups and operating conditions 
is included to evaluate the accuracy of the proposed method.

The paper is structured as follows: Section II focuses on the main phenomena that need to be taken into consideration for an accurate Li-ion battery modeling. Section III presents the mathematical formulation of the utilized algorithms. In Section IV, the main contribution of the paper, i.e. a more accurate hybrid SOC estimation solution, is presented. In Section $\mathrm{V}$, results are summarized together with the experimental validation, whereas information about the case studies and the simulation setup are given. Section VI concludes the paper.

\section{BATTERy MODEL}

Accurate battery modeling requires the incorporation of a number of phenomena, the most important of which are:

- The OCV-SOC relationship.

- The Hysteresis effect.

- Temperature and C-Rate impact.

- Capacity degradation.

A thorough discussion on these phenomena and their implication on battery modeling is presented in [17] and the need to identify the trade-off between good model accuracy and low complexity is addressed. Therefore, for brevity, only a brief presentation of them is given.

- The non-linear relationship between OCV and SOC is described by a piece-wise linear representation of each operation point of the battery [18] as expressed by (1)

$$
V_{\mathrm{OC}}=b_{0}+b_{1} \cdot \mathrm{SOC}
$$

where $b_{0}$ is the $y$-intercept and $b_{1}$ is the slope of the linear approximation.

- The hysteresis effect is expressed as voltage $V_{\mathrm{h}}$ in (2) and is incorporated as an additional state in the battery state-space model [19]

$$
\frac{d V_{\mathrm{h}}}{d t}=-\rho \cdot\left(\eta \cdot i_{\text {cell }}-v \cdot S_{\mathrm{D}}\right) \cdot\left[V_{\mathrm{hmax}}+\operatorname{sign}\left(i_{\text {cell }}\right) \cdot V_{\mathrm{h}}\right]
$$

where $v$ is a self-discharge multiplier for hysteresis expression, $S_{\mathrm{D}}$ is the self-discharge rate, $\eta$ is the coulombic efficiency, $V_{\text {hmax }}$ is the maximum hysteresis voltage, $\rho$ is the hysteresis parameter, which represents the convergence rate and $i_{\text {cell }}$ is the current flowing through the battery cell. In Li-ion batteries the self discharge is very low, therefore it can be ignored to reduce the model complexity.

- Temperature and C-rate impact are included during the real-time operation of the battery, by means of online parameter identification. Since battery parameters can vary due to temperature and C-rate impact as much as $800 \%$ [20], the SOC estimation can be affected significantly if they are neglected.

- The capacity degradation modeling is carried out using the AWTLS method [5]. It is an important parameter for SOC estimation using the CC method, thus it must be specified accurately.

Finally, the selection of the appropriate equivalent circuit model (ECM) is important, especially for online studies. The battery ECM of Fig. 1 is selected to simulate the battery operation and its electrical components are calculated using the FUDRLS method.

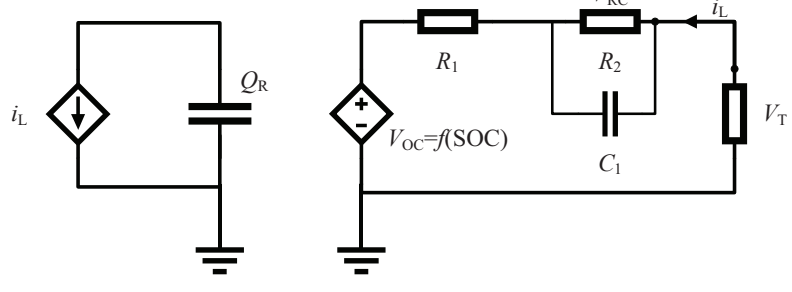

Fig. 1: Generic Li-ion battery cell equivalent circuit.

An increased number of RC branches may lead to higher accuracy, while on the other hand it will also increase the model complexity. Therefore, the number of the selected $\mathrm{RC}$ components, is a trade-off between model accuracy and complexity. Although in most cases, two RC branches are considered [21], it is proved that a single RC can result in sufficiently accurate results [9] if the excitation of the system is high [22], while the complexity is minimized. Further details on the state-space model of the ECM of Fig. 1 can be found in [5].

\section{Mathematical Formulation}

\section{A. Parameter Identification}

For the ECM of Fig. 1 the system transfer function equals:

$$
\frac{\mathbf{Y}(s)-b_{0}}{\mathbf{U}(s)}=\frac{R_{0} s^{2}+\left(\frac{b_{1}}{Q_{\mathrm{R}}}+\frac{1}{C_{1}}+\frac{R_{0}}{R_{1} C_{1} Q_{\mathrm{R}}}\right) s+\frac{b_{1}}{R_{1} C_{1} Q_{\mathrm{R}}}}{s\left(s+\frac{1}{R_{1} C_{1}}\right)}
$$

where $R_{0}, R_{1}, C_{1}$ and $Q_{\mathrm{R}}$ are the ECM parameters illustrated in Fig. $1 . Q_{\mathrm{R}}$ is the nominal capacity of battery pack/cell. Therefore, by using the voltage and current responses, battery parameters can be identified as well as their relation to aging and temperature, since the voltage output and the current includes this information.

Using the bi-linear transformation ( $\mathrm{s} \rightarrow \frac{2}{T} \frac{z-1}{z+1}$ ), we get the discrete transfer function (4) regarding sample time $T_{\mathrm{s}}$

$$
\frac{\left.\mathbf{Y}_{(} z^{-1}\right)-b_{0}}{\mathbf{U}\left(z^{-1}\right)}=\frac{x_{3}+x_{4} z^{-1}+x_{5} z^{-2}}{1+x_{1} z^{-1}+x_{2} z^{-2}}
$$

The coefficients of (4) are estimated by means of FUDRLS with variable forgetting factor (VFF) $\lambda$, using an alternative matrix form of the Bierman's equations in triangularization form. Factor $\lambda$ is updated by means of (5)-(6) based on [15].

$$
\begin{gathered}
\lambda(k+1)=1-\frac{v(k)}{\sigma_{0}^{2} \cdot N_{0}}, \quad \lambda_{\min } \leqslant \lambda \leqslant \lambda_{\max } \\
v(k)=\delta \cdot v(k-1)+(1-\delta) \cdot e(k)^{2}
\end{gathered}
$$

where $\delta$ is a weighting factor varying between $\lambda_{\min }$ and $\lambda_{\max }$, $e(k)$ is the prediction error, $v(k)$ is time-average expressions of $e(k)^{2}$ and $\sigma_{0}^{2}$ is the mean value of the error variance. $N_{0}$ is a constant that represents the system's memory.

Since the coefficients have been determined, the ECM parameters are calculated at each $T_{\mathrm{S}}$ based on (7)-(10).

$$
R_{0}=-x_{3}
$$




$$
\begin{gathered}
R_{1}=\frac{x_{2} x_{4}+x_{3} x_{2}^{2}+x_{5}}{\left(x_{2}-1\right)^{2}} \\
\tau=-\frac{T_{s}}{\ln x_{2}} \\
C_{1}=\frac{\tau}{R_{1}}
\end{gathered}
$$

Parameters identifiability and persistence of excitation are critical when identifying the ECM parameters. FUDRLS was chosen to address both issues and based on the comparative analysis presented in [23]. Note, that the FUDRLS algorithm is used to update $R_{0}, R_{1}$ and $C_{1}$, whereas $b_{0}$ and $b_{1}$ are updated based only on the SOC at a given time $t$.

\section{B. SOC Estimation}

SOC is an indicator of the present battery capacity and is used to regulate the charging/discharging process, while ensuring safe operation. Therefore, accurate SOC estimation is crucial for battery management systems (BMS). The main issues that arise in SOC estimation are the voltage inverse mapping, uncertainties in models, mapping nonlinearity, cycleto-cycle characteristics variations, and measurement errors [24]. The typical methods for online SOC estimation are:

1) CC method: This method is based on constant current measurements by computing the accumulated charge to estimate the SOC. This method is easy-to-implement and is suitable for online applications, thus is applied to several BMSs. However, CC method suffers from a long-term drift, due to measurement errors related to device sensitivity as well as errors depending on the integration process (numerical, trapeziodal etc.) [3]. Besides drifting, CC uses the previously measured capacity to calculate SOC, so if the battery capacity fades due to degradation, this may cause an additional SOC estimation error. Moreover, when the initial point of SOC is not known then the error might persist or even increase during battery operation. To improve the accuracy of the method, the SOC must be re-calibrated on a regular basis, such as resetting the SOC to $100 \%$ when the charger determines that the battery is fully charged or taking OCV after relaxation period. The CC method formulation is given by:

$$
\operatorname{SOC}\left(t_{2}\right)=\operatorname{SOC}\left(t_{1}\right)+\int_{t_{1}}^{t_{2}} \frac{\eta \cdot i(t)}{3600 Q_{\mathrm{R}}} d t
$$

where $\eta$ is the coulombic efficiency, $i(t)$ the current at time $t$, $Q_{\mathrm{R}}$ the capacity and $\mathrm{SOC}\left(t_{1}\right), \operatorname{SOC}\left(t_{2}\right)$ the $\mathrm{SOC}$ at $t_{1}, t_{2}$.

2) Model-based methods: Model-based methods use an electrical or electrochemical ECM to design an observer for real-time SOC estimation, presenting higher computational complexity than methods like CC. Most commonly used methods in this category are Kalman filter and its variants [25], [26], sliding-mode observer [27], smooth variable structure filter [28] to mention a few. These methods require accurate ECM parameters identification, which vary with temperature, aging and SOC of the battery cell. A state-space model is used to describe the battery system operation as shown in (12), in which the hysteresis effect is simulated by adding a hysteresis voltage as a state.

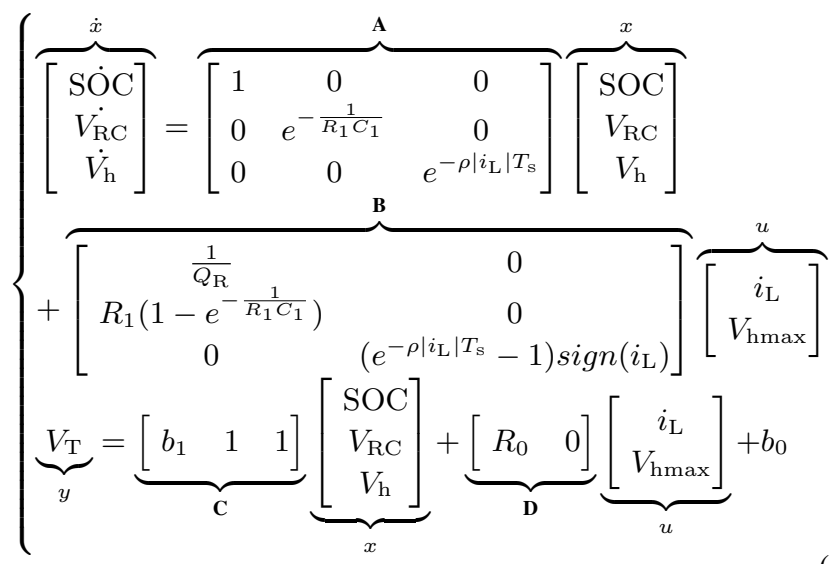

The state variables of the system are the SOC and the voltage drop on the RC branch. The unknown variables of the system are $R_{0}, R_{1}$ and $C_{1}$, while the known ones are $b_{1}$, $b_{0}$ and the nominal capacity $Q_{\mathrm{R}}$ that are extracted from the OCV-SOC curve and laboratory measurements, respectively.

Moreover, it is noteworthy that for Li-ion batteries that do not suffer from the hysteresis effect, a simpler model-based method that makes use of LKF and consists of a two-statespace model, as in [5], can be used.

3) OCV-based methods: Assuming that the output voltage of Li-ion battery equals to the OCV after long resting period, OCV can be calculated. Therefore, using look-up tables of OCV as a function of temperature and SOC, the battery SOC can be estimated. This method is easy-to-implement either in case the battery C-Rate is very small, since the relaxation effect is very small [7], or after long resting periods between cycling in order to reach balance. Although it is a simple method offering high precision, its main drawback is the need for long time intervals to reach an equilibrium. The time to reach from an operating state to a stable state depends on the SOC states and temperature. Experiments conducted at low temperatures revealed that $\mathrm{LiFePO}_{4}$ cell need more than two hours to reach equilibrium. Thus, such methods are applicable only when the battery is not operating for long periods.

\section{Proposed Hybrid Method Algorithm}

In this section, all modeling parts of the proposed technique are presented and a hybrid solution for SOC estimation is proposed. Modeling advantages and limitations of parts of the process are analyzed having as a goal to minimize the SOCestimation error. Special emphasis is given on the algorithms operation and their interactions.

The proposed hybrid scheme illustrated in Fig. 2 and model inputs, i.e. battery current and voltage signals are obtained from the corresponding sensors. The remaining battery capacity is estimated by applying AWTLS and is used to update the capacity both in the model-based and CC methods. ECM parameters are identified in order to prevent any abnormal operation of Li-ion battery. 


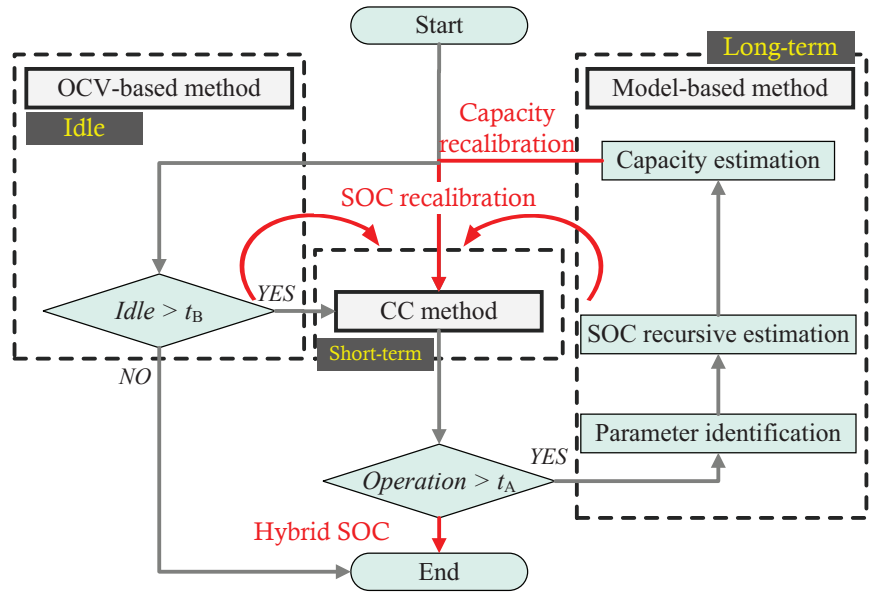

Fig. 2: Flow-chart representation of the proposed method.

SOC estimation is based on CC, model- and OCV-based methods and it is structured according to the following steps:

- Step 1. Short-term SOC estimation: use of CC.

- Step 2. Long-term SOC estimation: use of model-based methods, e.g. LKF to recalibrate SOC and solve the longterm drifting problem (for operation that exceeds $t_{\mathrm{A}}$ ).

- Step 3. SOC recalibration based on OCV-method for battery being idle for more than $t_{\mathrm{B}}$.

Note, that $t_{\mathrm{A}}$ and $t_{\mathrm{B}}$ refer to the timestamps at which the model- and OCV-based methods are activated, respectively.

\section{A. Short-term and Long-term SOC Estimation}

As mentioned above, regarding SOC estimation with modelbased methods, a combination of algorithms, e.g. FUDRLS and LKF needs to be applied. Consequently, the computational burden -especially for online applications- increases. Therefore, LKF can be used either after battery relaxation so that SOC estimation converges to its actual value or every $t_{\mathrm{A}}$ in order to solve the long-drifting problem of the CC method. Considering that by using the LKF a convergence to the actual value of SOC can be achieved within 2.5 minutes, a combination of a model-based solution and $\mathrm{CC}$ is proposed after an idle period of a battery and for three minutes. The convergence time depends on a number of parameters, such as the battery models' complexity, gains, and selected covariance matrices for difference applications. Therefore, although the proposed time is not expected to have a negative impact on the SOC initial error, such values need to be selected carefully. Since LKF attains SOC estimation, a trigger is used in order the corrected SOC, which is derived from the model-based method, to update the initial SOC of CC. Thus, the estimation error introduced by $\mathrm{CC}$ to the initial SOC value is eliminated.

LKF-based SOC estimation is almost independent on the capacity degradation, considering that battery-cells assumed as dead when the capacity has faded $20 \%$ from its nominal value [5]. The reason is that the solution of the Riccati equation in a time invariant system converges to steady state co-variance if the matrices $\mathbf{A}, \mathbf{C}$ of (12) are observable. Note, that the SOC estimation accuracy depends on the parameter identification and hysteresis voltage estimation.
Afterwards, CC is utilized for SOC estimation in order to benefit from the lowest possible computational burden. As mentioned in Section III, CC offers great accuracy and very low estimation error during a period of time before the longterm drift problem appears. Since, the time at which this phenomenon appears is not well determined in the literature, LKF is reactivated at every $t_{\mathrm{A}}$ to assist SOC convergence to its actual value. Reactivation of LKF has been decided every $60 \operatorname{mins}\left(t_{\mathrm{A}}=60 \mathrm{~min}\right)$ after a trial and error approach, since it depends on the BESS technology and is operation specific. Typical values for $t_{\mathrm{A}}$ are $>30$ mins. As highlighted in [17], LKF can be used not only for robust SOC estimation but also for auto-correction of the SOC estimation process as well, even under extreme measurement errors. Therefore, the periodic utilization of LKF is proposed as a solution that ensures accurate SOC estimation but does not burden the operation.

Note, that LKF introduces high estimation error for battery operation within the areas that OCV-SOC curve presents high non-linearity, i.e., before $15 \%$ and after $85 \%$ of SOC. Thus, for battery operation in these areas, the $\mathrm{CC}$ method should be used for SOC estimation.

\section{B. SOC Recalibration}

Since an OCV-based method is very accurate when the battery is not operating for long time periods ( $t_{\mathrm{B}}$ usually exceeds 45 mins and depends on battery type), it can be used for SOC recalibration. When the battery does not operate on-load for more than $t_{\mathrm{B}}$, voltage equals to $\mathrm{OCV}$ and by using the OCV-SOC look-up tables, the SOC can be extracted. Therefore, the initial point of SOC in CC method can be updated. In this way the initial SOC point in CC method is known and the estimation problem that $\mathrm{CC}$ adds to the overall model is overcome. In case the battery is idle for a period of time shorter than $t_{\mathrm{B}}$, LKF is preferred to implement SOC estimation for on-load battery operation [29].

\section{Interaction with capacity estimation $C C \& A W T L S$}

To estimate capacity degradation, an AWTLS algorithm is used [16]. The algorithm identifies the slope of the equation $y=Q_{R} \hat{x}$, where $x=\operatorname{SOC}\left(\mathrm{t}_{2}\right)-\operatorname{SOC}\left(\mathrm{t}_{1}\right)$ and $y=$ $\int_{t_{1}}^{t_{1}} \frac{\eta i(t)}{3600} d t$. It is assumed that $\eta=1$ at all current values and temperature within normal operating conditions. Literature [5], [16] indicates that AWTLS offers accurate results regarding the remaining capacity of the battery. Note, that the selected algorithm for the model-based method is independent of the capacity degradation [5]. Therefore, the SOC step that is used in AWTLS algorithm is updated by the estimated SOC of LKF.

AWTLS is considered as an accurate capacity estimation method, since the estimation error is in the range of $5 \%$ or lower [16]. AWTLS requires only simple mathematical operations, is computed in a recursive manner and its coefficients are updated only when additional data points become available. The method is superior to the other TLS methods since it allows individual weighting on SOC and current data points, it gives bounded estimation error and is robust to different operating conditions. Furthermore, CC typically introduces an estimation error, due to the strong relation to the remaining 
capacity of the battery as it can be seen in (11). Therefore, the capacity needs to be estimated and systematically updated in order to improve the accuracy of the CC method and also overcome the limitations of both algorithms.

The main drawback of AWTLS, for online capacity estimation, is the introduction of a fluctuation when it comes to update the remaining capacity. Assuming that the results of AWTLS are not filtered, the SOC estimation error will be increased, since the capacity fluctuation will add noise to the SOC estimation results. Therefore, a moving average (MA) filter is suggested to calculate the average value of the data during a specific time window. The window is of fixed length and slides by one sample as each new measurement point is received. At each step the filter calculates the new value.

$$
Q_{\mathrm{R}, \mathrm{rem}}=\frac{1}{M} \int_{t}^{t+M} Q_{\mathrm{R}} d t
$$

where $Q_{\mathrm{R}, \mathrm{rem}}$ is the MA-smoothed estimated capacity, $Q_{\mathrm{R}}$ is the estimated capacity derived from AWTLS and $M(M=20)$ is the length of the time window. The value of $M$ has been chosen after conducting a trial and error approach. Having estimated $Q_{\mathrm{R}, \mathrm{rem}}$, the nominal capacity value used in $\mathrm{CC}$ formulation of (11) is updated and replaces $Q_{\mathrm{R}}$. As a result $\mathrm{CC}$ becomes more accurate during battery lifetime and does not increase the error when there is a significant capacity degradation.

Note, that the algorithms for AWTLS and FUDRLS are presented in detail, i.e. mathematical expressions, coefficients and initialization, in [16] and [15], respectively.

\section{RESUltS \& EXPERIMENTAL VALIDATION}

In this section, simulation results as well as experimental validation for all modeling parts, are provided. Emphasis is given on the SOC estimation results and the improved accuracy that can be achieved by the proposed hybrid solution when compared to the conventional SOC estimation methods. Extreme operating conditions, such as high C-Rate and temperature, were not examined in this work although they are expected to affect the accuracy of SOC estimation, since in such extraordinary cases the BMS is expected to intervene providing protection for the equipment.

\section{A. Simulation Setup \& Case Studies}

To verify the accuracy and efficiency of the proposed model four test setups, i.e., Test 1, 2, 3 and 4 were carried out. For all tests, measurements of voltage $V$ and current $I$ were recorded. Using the measured $V$ and $I$ responses the battery states of (12) are estimated. Tests were performed at varying temperatures and different $\mathrm{C}$-Rates while the sample time was selected equal to $0.5 \mathrm{~s}$ [5]. The LifeTest SBT0550 battery cell tester of PEC Corporation, offering very accurate current measurements at a rate of $1 \mathrm{~ms}$, was used. The current measurement was used to integrate current for CC-based SOC estimation and served as a reference for SOC estimation.
1) Test 1: The first test case, refers to the supply of an electric-vehicle profile. A Li-ion battery cell with LTO/Mixed Oxide chemistry is used. The load profile in terms of measured current and voltage is shown in Figs. $3 \mathrm{a}$ and $3 \mathrm{~b}$, respectively.

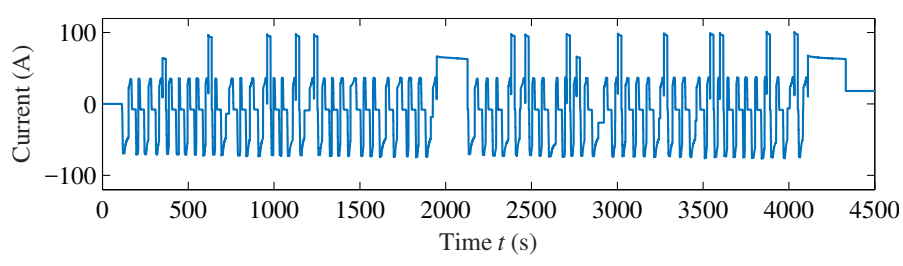

(a) Load-profile (current) for Test 1 .

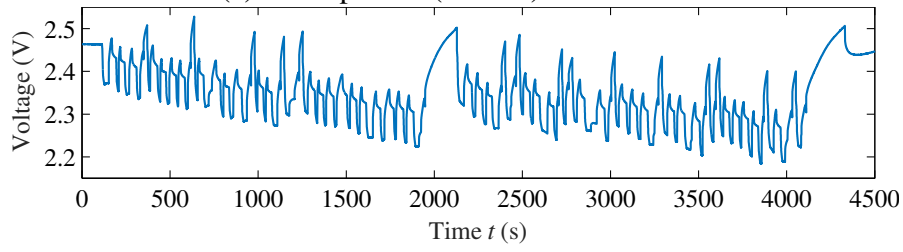

(b) Load-profile (voltage) for Test 1.

Fig. 3: Load-profile (current \& voltage) for Test 1.

2) Test 2: A dynamic load profile is used to examine the SOC estimation accuracy, while cycling Li-ion in a midrange of SOC with high C-rates. A Li-ion battery cell with Graphite/LMO-Spinel chemistry is operated and the measured current and voltage profiles are presented in Figs. $4 \mathrm{a}$ and $4 \mathrm{~b}$.

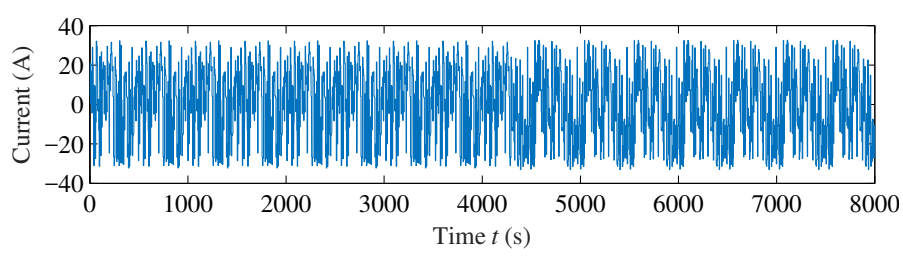

(a) Load-profile (current) for Test 2 .

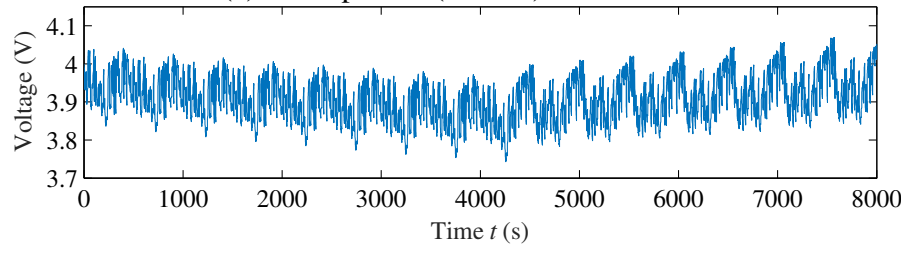

(b) Load-profile (voltage) for Test 2.

Fig. 4: Load-profile (current \& voltage) for Test 2.

3) Test 3: Figures 5a and 5b refer to the current and voltage profiles of a pulse test, in which $10 \mathrm{~s}$ and $30 \mathrm{~s}$ pulses are repeated at $10 \%$ SOC increments. The same LMO battery cell from Test 2 was used. A complete charge and discharge of the battery is performed to validate the performance of the hybrid method. After any discharging step of $10 \%$ of SOC, battery remains idle for 45 mins. As a result, the OCV-based method is triggered to recalibrate the SOC.

4) Test 4: Figures $6 a$ and $6 \mathrm{~b}$ correspond to the current and voltage profile of a test which was performed to validate the effect of the capacity fade on the SOC estimation. The 


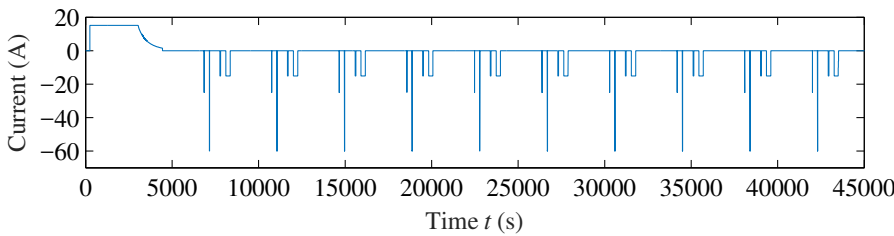

(a) Load-profile (current) for Test 3.

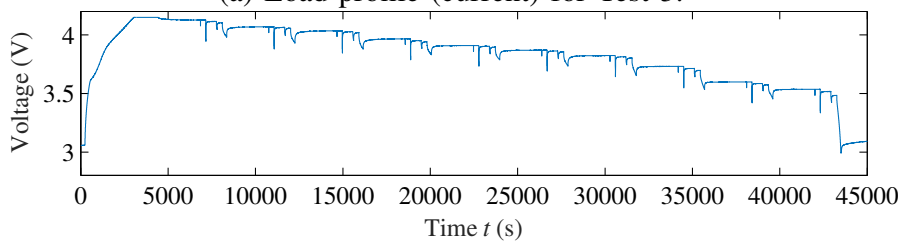

(b) Load-profile (voltage) for Test 3.

Fig. 5: Load-profile (current \& voltage) for Test 3.

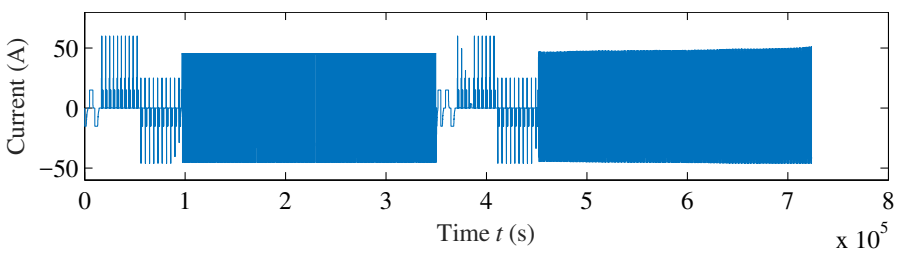

(a) Load-profile (current) for Test 4.

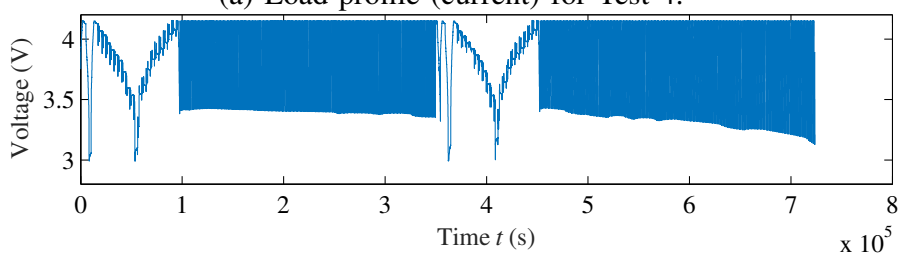

(b) Load-profile (voltage) for Test 4.

Fig. 6: Load-profile (current \& voltage) for Test 4.

LMO battery used for Tests 2 and 3 was also adopted. The experiment consists of a complete discharge and charge of the battery, gradual discharge by $10 \%$ of SOC until full discharge of the battery, step-by-step charge by $10 \%$ of SOC until full charge and cycling of the battery between $80 \%$ and $20 \%$ of SOC with a $3 \mathrm{C}$ C-Rate.

\section{B. Parameter Identification \& Capacity Estimation}

Concerning parameter identification, the results of $R_{0}, R_{1}$ and $C_{1}$ for Test 1 can be found in [23], while the respective ones for Test 2 are summarized in Fig. 7. For brevity, results for Tests 3 and 4 are not presented. As illustrated in [15], [23], using FUDRLS algorithm for the parameter identification improves SOC estimation accuracy of model-based methods. This occurs due to the fact that FUDRLS provides fast convergence to the real values. As a result by updating the internal parameters of the state-space model used in LKF, the accuracy of the model based method increases.

The results for the capacity estimation of the LMO battery cell can be seen in Fig. 8. The rms estimation error is approximately $2 \%$. As for the LTO battery cell due to the

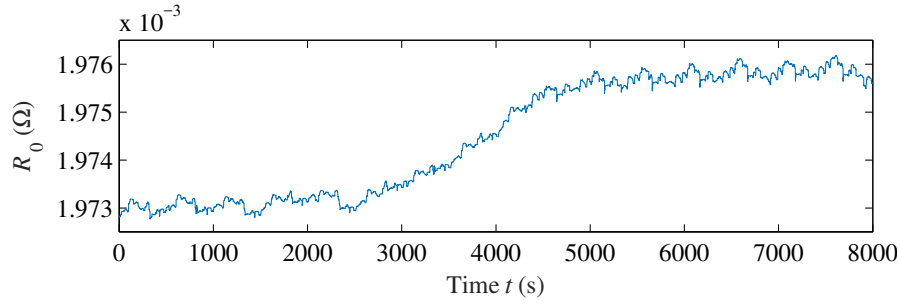

(a) Battery cell - $R_{0}$ identification.

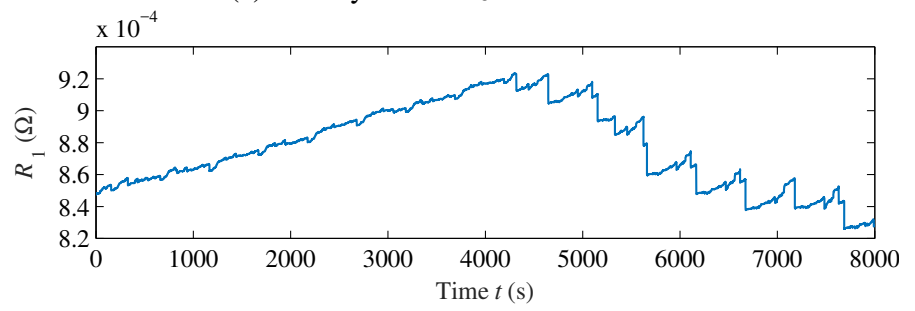

(b) Battery cell - $R_{1}$ identification.

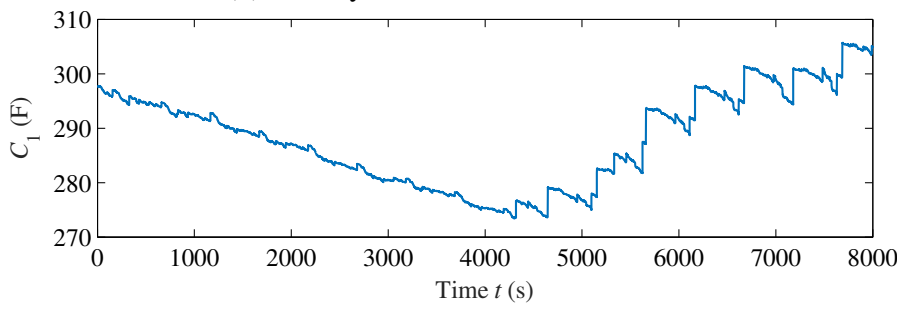

(c) Battery cell - $C_{1}$ identification.

Fig. 7: Parameter identification with FUDRLS for Test 2.

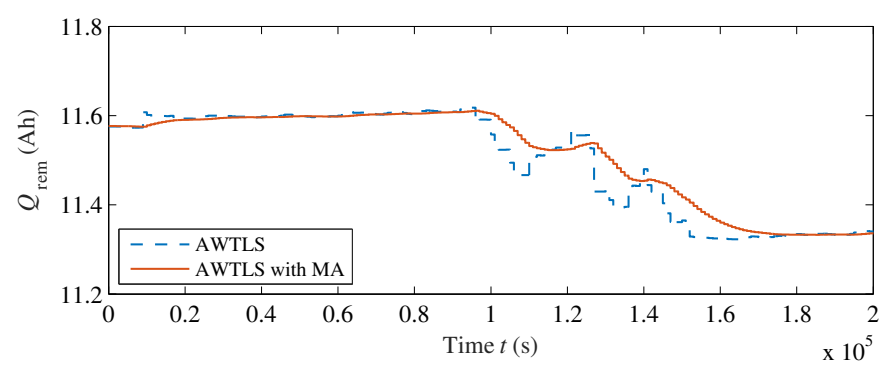

Fig. 8: Battery cell - capacity estimation.

cell chemistry no capacity drop during the experiment is observed. The capacity remained constant and for brevity is not presented. Capacity estimation results can be found in [5]. Results that last up to $20000 \mathrm{~s}$ are presented due to the battery type. An experiment that lasts significantly longer than expected is needed in order capacity degradation to be evident.

Although the 0.4 Ah capacity drop - from 11.6 to $11.2 \mathrm{Ah}$ looks negligible, such a drop within only nine operation cycles corresponds to a $2.6 \%$ drop of the nominal battery capacity, which is significant when considering that there are applications for which a $20 \%$ drop could lead to battery replacement. To achieve such an intense drop, extreme charging/discharging conditions within a range of $-4 \mathrm{C}$ to $4 \mathrm{C} \mathrm{C}$-Rates were imposed in the experiments. 


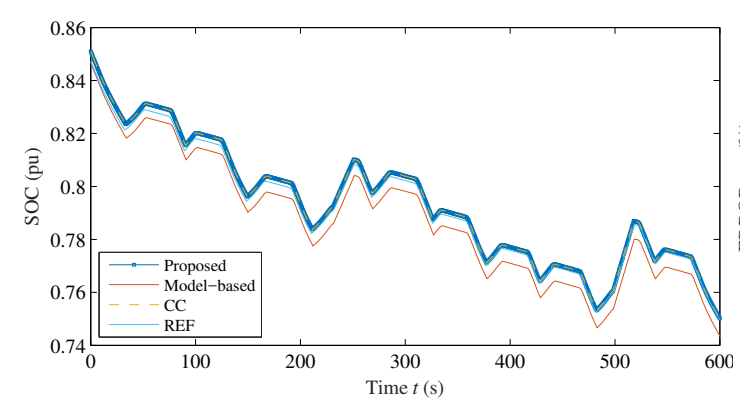

(a) Cycle1 - SOC estimation.

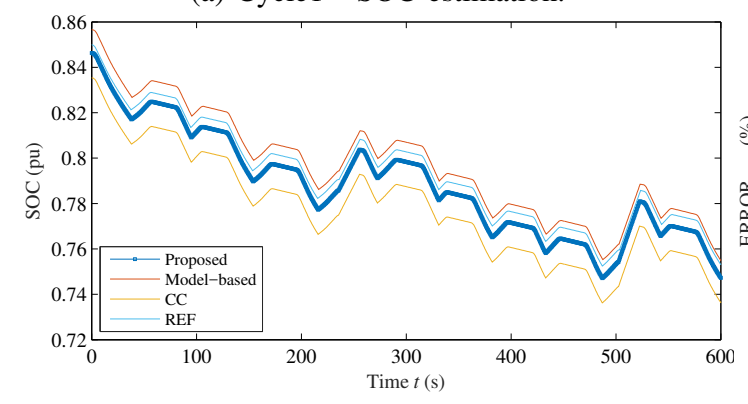

(d) Cycle9 - SOC estimation.

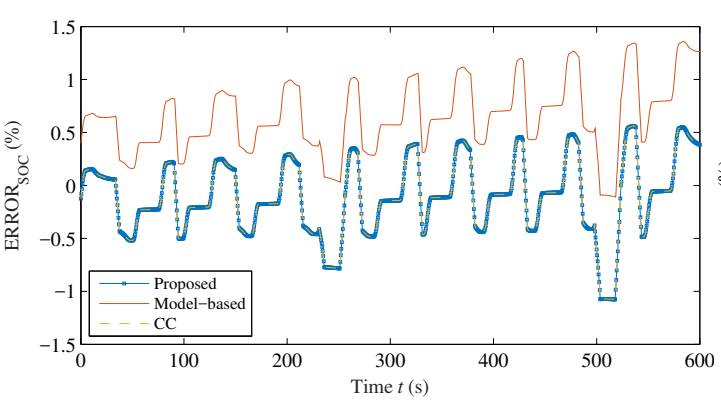

(b) Cycle1 - SOC estimation error.

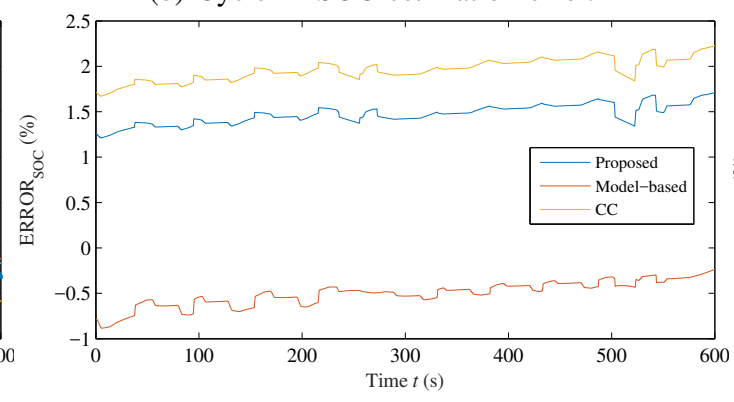

(e) Cycle9 - SOC estimation error.

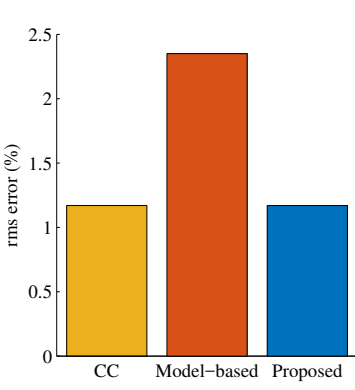

(c) Cycle 1 - rms error.

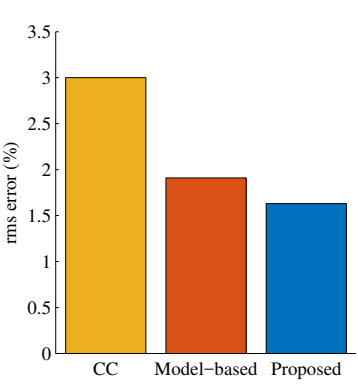

(f) Cycle9 - rms error.

Fig. 9: Test 1: Summarized SOC estimation results for Cycle1 and Cycle9.

\section{Short-term and Long-term SOC Estimation}

SOC reference is extracted by using the CC method with a sample time equal to $1 \mathrm{~ms}$, to minimize the SOC estimation error. Details on SOC reference extraction can be found in [17]. The efficiency of the hybrid model is examined while the battery used for Test 1 completes 9 cycles of the load profile that is presented in Figs. 3a and $3 \mathrm{~b}$ and while the battery used for Test 2 completes 16 cycles of the load profile that is presented in Figs. 4a and 4b, respectively.

In Fig. 9, results regarding SOC estimation and the rms error, for Test 1, are summarized. Results considering the first and ninth cycle are presented in Figs. 9a-9c and Figs. 9d9f, respectively. Likewise in Fig. 10, results for Test 2 are summarized, for the first cycle (Figs. 10a-10c) or cycle 16 (Figs. 10d-10f) of the respective battery. To perform a SOC estimation error assessment, the root mean square (RMS) error metric is deployed. RMS error is a quadratic scoring rule that measures the average magnitude based on the following formulation:

$$
\mathrm{RMS}_{\text {error }}=\sqrt{\frac{1}{n} \sum_{j=1}^{n}\left(y_{j}-\hat{y}_{j}\right)^{2}}
$$

where $y_{j}$ is the reference and $\hat{y}_{j}$ the estimated value and $n$ the number of sample data.

SOC estimation accuracy comparative results, between the CC method, LKF and the proposed hybrid implementation, are illustrated in the bar-graphs in terms of the rms error. As expected, at the beginning of both batteries cycling the rms error in case of the CC method is the lowest, i.e. in the range of approximately $1 \%$. Therefore, in Figs. 9a, 9b, 10a and 10b, CC and Hybrid curves overlap. However, after some cycles, the estimation accuracy of the CC method decreases, presenting an rms error of approximately 4\%, making it difficult to track the battery SOC correctly.

On the other hand, although model-based methods present higher SOC estimation error compared to the $\mathrm{CC}$ method at the beginning of the batteries cycling, they can track SOC more accurately after a few cycles. From Figs. 9f and 10f, it is evident that the SOC estimation of the LKF method improves with cycling operating time and becomes more accurate compared to the $\mathrm{CC}$ method when more cycles are reached.

As presented in Figs. 9 and 10, exploitation of both methods advantages by means of the hybrid implementation (blue color) can lead to more accurate SOC estimation over battery lifetime. The rms SOC estimation error of the proposed hybrid solution is analyzed in Figs. 9c, 9f, 10c and 10f. It is illustrated that at the beginning of cycling, results are close to the corresponding of the CC method, while for higher cycling times, the proposed solution outperforms the others. As expected, since the SOC initialization is very accurate and contains minimum error, in short-term, CC outperforms model-based alternatives, whereas the opposite behavior occurs for long-term. Note that the noise level in measurements of both Figs. 9 and 10, is typically four orders of magnitude smaller than the reference current value, therefore it cannot be seen in the plots.

In Fig. 11, the influence of the remaining capacity update on the SOC estimation, in case of Test 2, is documented. Note, that SOC estimation is conducted using the proposed method. Given a $3.2 \%$ battery capacity degradation, the SOC estimation is more accurate by $2.14 \%$ when the remaining capacity is updated. This difference between SOC estimation results with or without updating of the remaining capacity will be even higher if battery deterioration reaches $20 \%$, i.e. the life expectancy of a Li-ion battery. 


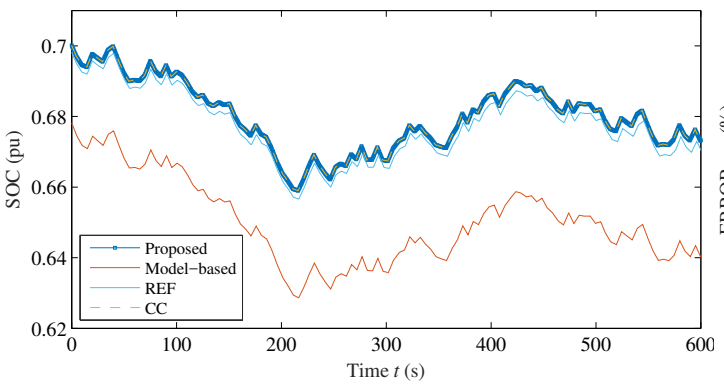

(a) Cycle1 - SOC estimation.

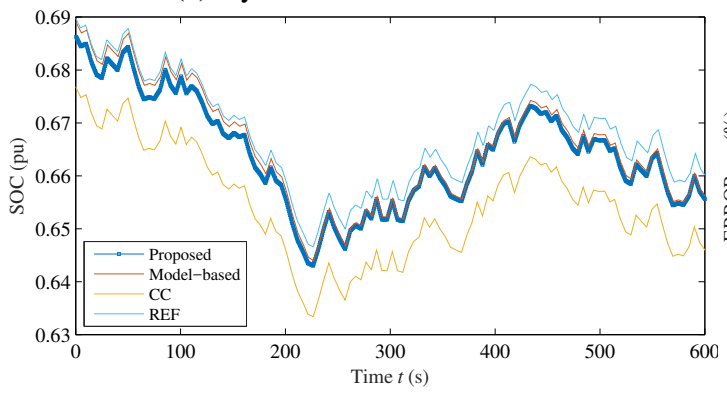

(d) Cycle16 - SOC estimation.

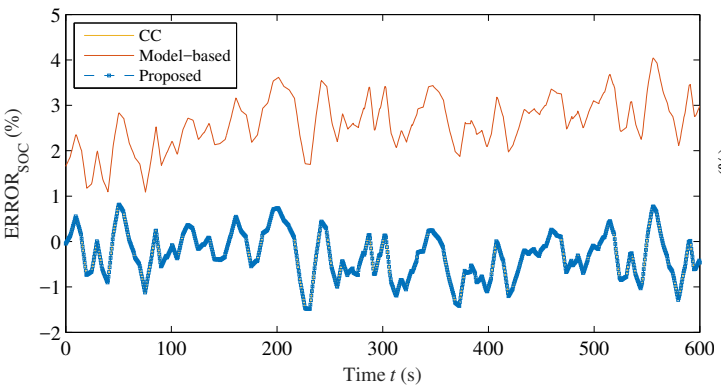

(b) Cycle1 - SOC estimation error.

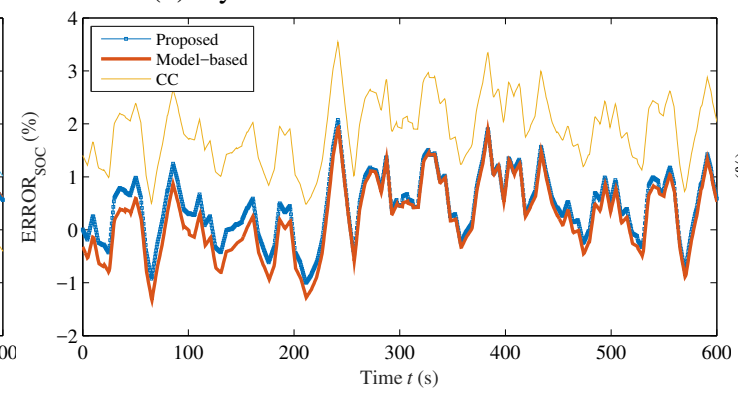

(e) Cycle16 - SOC estimation error.

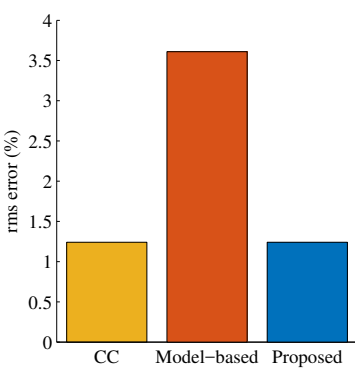

(c) Cycle1 - rms error.

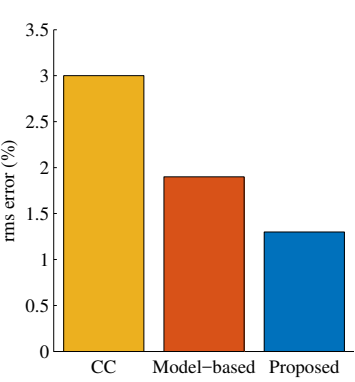

(f) Cycle16 - rms error.

Fig. 10: Test 2: Summarized SOC estimation results for Cycle1 and Cycle16.

In Figs. 12a and 12b, SOC estimation results regarding Test 4 are presented, to highlight the effects of capacity fade on SOC estimation. Fig. 12a refers to capacity estimation. Considering a linear interpolation from 13.754 to $11.818 \mathrm{Ahs}$, the estimation error is $<5 \%$ for the time range between 0 and $3.5 \cdot 10^{5} \mathrm{~s}$. However, as the algorithm gets more information for the $\Delta \mathrm{Q}$ and $\Delta \mathrm{SOC}$ (update of the value), the AWTLS algorithm performs better and the estimation accuracy increases. Thus, the estimation error between $3.5 \cdot 10^{5}$ and $7.25 \cdot 10^{5} \mathrm{~s}$ is reduced to less than $2.5 \%$.

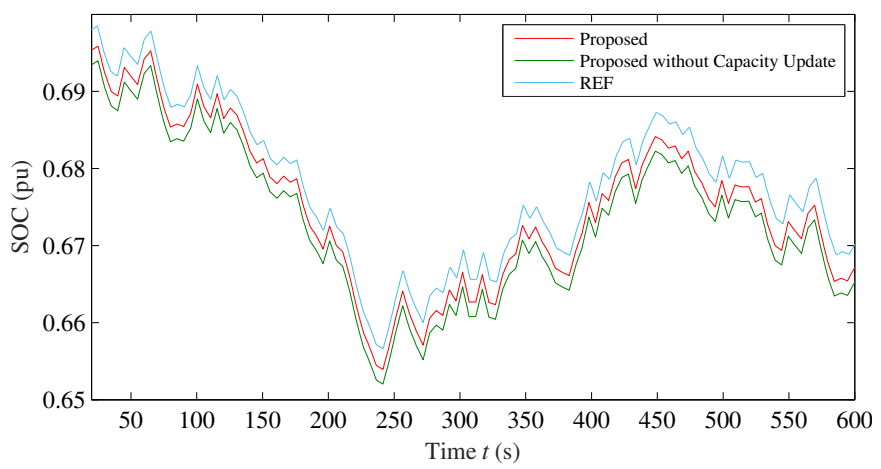

Fig. 11: SOC estimation and update of the remaining capacity.

In Fig. 12b, SOC estimation compared to reference SOC is presented for the last charging and discharging pulse, before the end of the experiment. A comparison between the scheme in which AWTLS is used to update the capacity of the battery of the hybrid model and the scheme in which AWTLS is not utilized is depicted. The estimation performance of the hybrid model is improved when the remaining capacity is updated. The error for the proposed approach is $2.32 \%$, while in case the capacity is not updated equals $10.87 \%$.

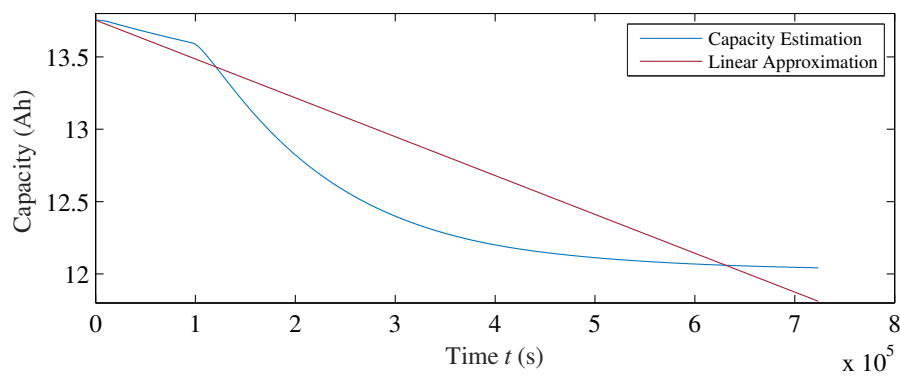

(a) Capacity estimation for Test 4 .

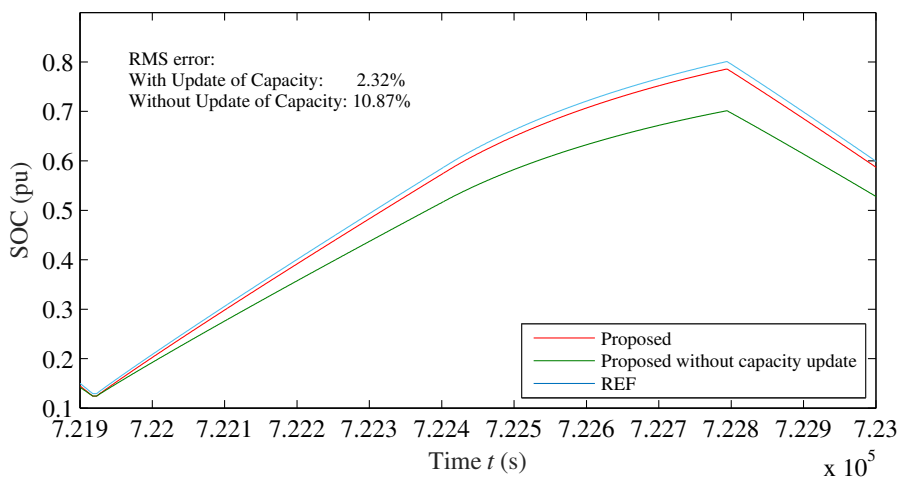

(b) SOC estimation and update of the remaining capacity.

Fig. 12: Test 4: Capacity and SOC estimation results.

A literature review [30]-[33] on alternative algorithms for online capacity estimation revealed the results that are summarized in Table I and proved that the proposed methodology presents highly accurate capacity estimation results as well.

When a model-based method is used for SOC estimation an error in the range of 2-3\% is always expected and depends on 
TABLE I: Capacity Estimation Algorithms’ Evaluation

\begin{tabular}{c|ccc} 
Ref. & Algorithm & Cell type & Error \\
\hline$[30]$ & 2 EKFs & Li-NMC & $3.00 \%(\max )$ \\
{$[31]$} & Double EKF & Li-ion & $5.00 \%(\max )$ \\
{$[32]$} & Multi-scale EKF & LiPB & $3.93 \%(\operatorname{ms})$ \\
{$[33]$} & Neural Network & Ni-MH & $2.67 \%(\max )$
\end{tabular}

the non-linear electrochemical dynamics of the battery cells. Moreover, there are several factors that affect the accuracy of the CC method including temperature, battery history (number of cycles, depth of discharge during the cycles), discharge current etc., whereas the estimation error of $\mathrm{CC}$ is increasing after 10 cycles. If there are no corrective actions, the $\mathrm{CC}$ based SOC estimation error can reach $9 \%$. To that end and in order to reduce that estimation error, the proposed hybrid combination can be utilized.

\section{SOC Recalibration}

As already mentioned, both model-based [17] and OCVbased methods [29] can be utilized for SOC recalibration and to maintain SOC within accepted estimation error limits. Therefore, based on the proposed implementation, their periodic triggering can assist to more accurate results, while not affecting the computational burden significantly.

Under normal operating conditions, i.e. for temperatures higher than $10{ }^{\circ} \mathrm{C}$, the OCV-SOC relationship is static over battery lifetime and does not vary significantly. Therefore, after a long period during which battery remains idle equal to $t_{\mathrm{B}}$, a trigger can be used to recalibrate SOC and minimize the SOC estimation error. Test 3 can be used, since the battery remains idle for a long period after each discharge step, i.e. for $t_{\mathrm{B}}=$ 45 mins. The impact of the OCV-based method would have been more significant in case idle periods were even longer.

In Fig. 13, full-range (from 0 to $100 \%$ ) SOC estimation results are summarized. More specifically, Fig. 13a illustrates how the proposed hybrid implementation tracks more accurately than the stand-alone methods the reference SOC while considering a $20 \%$ SOC initialization error. Moreover Figs. $13 \mathrm{~b}$ and $13 \mathrm{c}$ indicate the timestamps at which either the modelbased or the OCV-based methods were triggered.

First, it is clear that there is a high SOC estimation error above 0.9 and below 0.1 of SOC that is caused by the high non-linearity and the low excitation that the battery system presents within these regions. As a result, the modelbased method becomes less accurate, and thus the estimation accuracy of the hybrid method is negatively affected. However, between 0.1 and 0.9 of SOC, the estimation accuracy of the hybrid method is high due to the better performance of the model-based method. Moreover, since the OCV-based method is triggered, the estimation error becomes almost zero. Especially within the range of 0.35 and 0.75 of SOC, where the rms estimation error of the model-based method is below $3 \%$, the estimation accuracy of the hybrid method is well improved. As a result, it is proposed to trigger the model-based method when the range of SOC is within such values. Finally, activation of the OCV-based method when the battery is idle for a long period, results in limiting the estimation error to

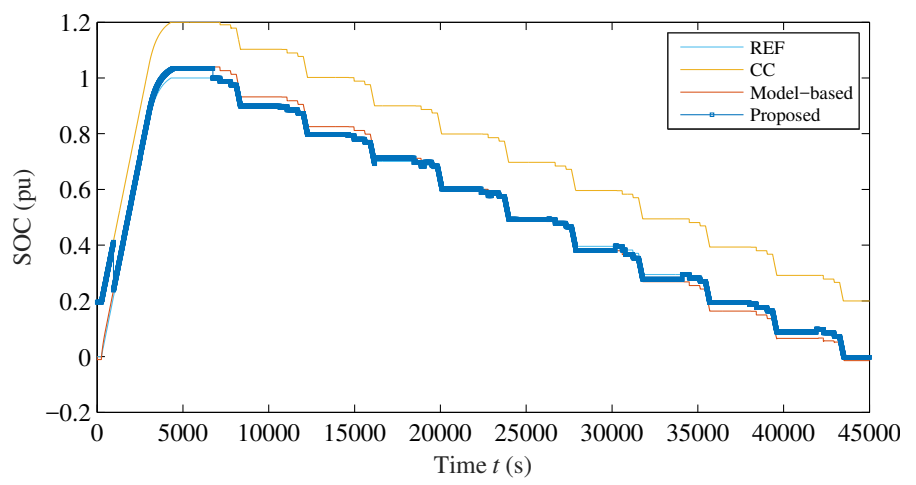

(a) SOC estimation - comparative results.

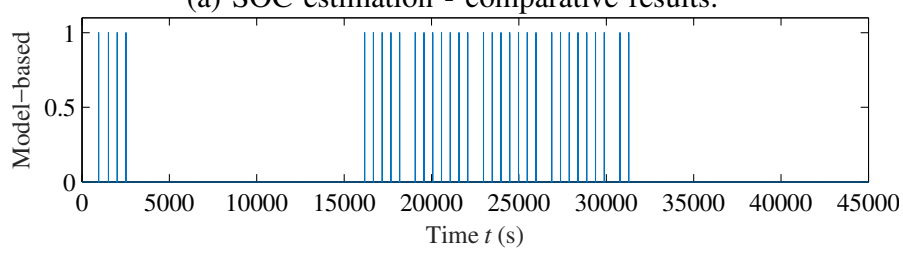

(b) Triggering of model-based SOC estimation.

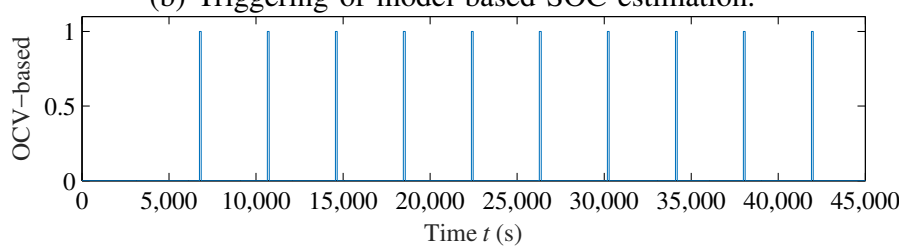

(c) Triggering of OCV-based SOC estimation.

Fig. 13: Test 3: Summarized SOC estimation results.

less than $1 \%$. The time interval, in which the battery remains idle, until the OCV-based method is triggered depends on the battery technology (how much time is needed for the OCV to become equal to the voltage, when the battery is idle).

Note also, that the $\mathrm{CC}$ method never converges to the actual SOC value and presents a persistent estimation error. On the other hand, the model-based method can track the actual SOC value a few seconds after the simulation starts. This leads to the error correction, achieved by the hybrid method (in the first seconds). Then, when the OCV-based method is activated the estimation error is further reduced to less than $1 \%$. During idle periods, the OCV-based is triggered with a constant frequency, since the idle periods are of equal length.

\section{Discussion - Extension to BAtTery PACKS}

The proposed solution can be also applied to battery packs connected either in series or in parallel. Battery cells/packs are simulated as black boxes and especially in the case of modelbased methods application, the measured voltage and current signals are used as inputs to estimate the SOC and the battery capacity to identify the model parameters.

Compared to cell-level modeling, the main difference in battery pack level modeling refers to the application of modelbased methods for SOC estimation and mainly on the extraction of the OCV-SOC curve. More specifically, in case of a battery pack more SOC breakpoints are required, since more 
nonlinear parts through the OCV-SOC curve exist that need to be linearized. Therefore, the modeling accuracy depends significantly on the extraction of the OCV-SOC curve and the selection of the SOC breakpoints.

Note, that the proposed method can be applied to each cell of a battery pack in case voltage and current sensors exist, by extracting the OCV-SOC curve for each individual cell. Although, the application of the proposed method to each cell might increase the SOC estimation accuracy, it will also affect the computational burden, accordingly. Since computational burden is always an issue with real-time applications, the impact of the hybrid approach to that end was evaluated for Test 3 by examining the computational time as well as the CPU usage. Three simulations have been conducted for SOC estimation, namely, CC, model-based and the hybrid method and their comparison results are summarized in Table II, proving that the proposed approach does not result in unnecessary increase of computational burden.

\section{TABLE II: Computational Burden Evaluation}

\begin{tabular}{c|cc} 
Method & CPU usage increase (\%) & Simulation time $(\mathrm{s})$ \\
\hline CC & 11 & 0.56 \\
Model-based & 19 & 1.24 \\
Hybrid & 17 & 1.08
\end{tabular}

For longer battery operation periods, the performance improvements of the proposed scheme will be far more evident.

\section{CONCLUSIONS}

In this paper, an improved solution for the SOC estimation process of $\mathrm{Li}$-ion batteries is presented. The proposed hybrid approach combines the advantages of three different SOC estimation approaches and provides more accurate SOC estimation.

The proposed approach can be used for real-time applications of Li-ion batteries that require fast actions and accurate SOC estimation. Although in such applications, usually CC method is utilized because of its short-term accuracy and its simplicity, the proposed hybrid solution can be used instead, in order to provide accurate results over battery lifetime without increasing the computational burden as model-based solutions like LKF do, when operated for both short and long term operation. Each stand-alone approach contributes significantly depending on the need for short- or long-term SOC estimation as well as on the need for SOC recalibration. Moreover, the proposed solution can be attractive for extended battery packs, where SOC estimation is even more important than in case of a simple battery cell. Finally, it is noteworthy that the proposed hybrid methodology is expandable and non-specific, since alternative algorithms and different ECM with more RC branches can be considered.

For future work an intelligent machine learning technique can be developed in order to control the recalibration of SOC initial point (moment of triggering) that occurs when OCVbased and model-based methods are used in order to minimize the estimation error. The impact of the time parameters $t_{\mathrm{A}}$ and $t_{\mathrm{B}}$ on the computational burden and the estimation accuracy can also be assessed. Finally, additional research on technoeconomic level can be conducted to point out what are the implications with the computational burden and what are the economical benefits when a hybrid approach for SOC estimation is deployed.

\section{ACKNOWLEDGEMENT}

The authors are thankful to ABB Corporate Research Center in Västerås, Sweden, for providing the necessary laboratory facilities to test a series of batteries under varying conditions. They would also like to thank Tomas Tengnér, global product specialist for energy storage at ABB Marine Applications unit in Billingstad, Norway, for insightful advice on the latest energy storage developments.

\section{REFERENCES}

[1] D. I. Doukas, P. Bakas, A. Marinopoulos, and H. Kim, "Energy storage integration into grid connected utility-scale photovoltaic (PV) systems," in 2013 IEEE Grenoble Conference, Jun. 2013, pp. 1-6.

[2] H. Rahimi-Eichi, U. Ojha, F. Baronti, and M. Y. Chow, "Battery management system: An overview of its application in the smart grid and electric vehicles," IEEE Ind. Electron. Mag., vol. 7, no. 2, pp. 4-16, Jun. 2013.

[3] K. W. E. Cheng, B. P. Divakar, H. Wu, K. Ding, and H. F. Ho, "Batterymanagement system (BMS) and SOC development for electrical vehicles," IEEE Trans. Veh. Technol., vol. 60, no. 1, pp. 76-88, Jan. 2011.

[4] A. R. F. G. D. Zhou, K. Zhang and A. Miraoui, "Parameter sensitivity analysis for fractional-order modeling of lithium-ion batteries," Energies, vol. 9, no. 3, 2016 .

[5] G. S. Misyris, T. Tengnér, A. G. Marinopoulos, D. I. Doukas, and D. P. Labridis, "Battery energy storage systems modeling for online applications," in IEEE Manchester PowerTech, Jun. 2017, pp. 1-6.

[6] K. S. Ng, C. S. Moo, Y. P. Chen, and Y. C. Hsieh, "Enhanced Coulomb counting method for estimating state-of-charge and state-of-health of Lithium-ion batteries," Appl. Energy, vol. 86, no. 9, 2009.

[7] M. A. Roscher and D. U. Sauer, "Dynamic electric behavior and open-circuit-voltage modeling of $\mathrm{LiFePO}_{4}$-based Lithium ion secondary batteries," J. Power Sources, vol. 196, no. 1, pp. 331-336, 2011.

[8] L. Lu, X. Han, J. Li, J. Hua, and M. Ouyang, "A review on the key issues for Lithium-ion battery management in electric vehicles," J. Power Sources, vol. 226, pp. 272-288, 2013.

[9] X. Hu, S. Li, and H. Peng, "A comparative study of equivalent circuit models for Li-ion batteries," J. Power Sources, vol. 198, p. 359, 2012.

[10] M. Hannan, M. Lipu, A. Hussain, and A. Mohamed, "A review of lithium-ion battery state of charge estimation and management system in electric vehicle applications: Challenges and recommendations," Renew. Sustain. Energy Rev., vol. 78, pp. 834 - 854, 2017.

[11] G. L. M. S. D. I. S. A. I. S. J. Meng, M. Ricco and R. Teodorescu, "An overview and comparison of online implementable SOC estimation methods for lithium-ion battery," IEEE Trans. Ind. Appl., vol. 54, no. 2, pp. 1583-1591, Mar. 2018.

[12] Q. Y. H. H. R. Xiong, J. Cao and F. Sun, "Critical review on the battery state of charge estimation methods for electric vehicles," IEEE Access, vol. 6, pp. 1832-1843, 2018.

[13] A. R. F. G. D. Zhou, K. Zhang and A. Miraoui, "Online estimation of lithium polymer batteries state-of-charge using particle filter-based data fusion with multimodels approach," IEEE Trans. Ind. Appl., vol. 52, no. 3, pp. 2582-2595, May 2016.

[14] F. G. D. P. A. M. D. Zhou, A. Ravey and K. Zhang, "Online estimation of state of charge of li-ion battery using an iterated extended kalman particle filter," in 2015 IEEE Transport. Electrific. Conf. and Expo (ITEC), Jun. 2015, pp. 1-5.

[15] T. Kim, Y. Wang, Z. Sahinoglu, T. Wada, S. Hara, and W. Qiao, "Fast UD factorization-based RLS online parameter identification for modelbased condition monitoring of lithium-ion batteries," in 2014 American Control Conference, Jun. 2014, pp. 4410-4415.

[16] G. L. Plett, "Recursive approximate weighted total least squares estimation of battery cell total capacity,' J. Power Sources, vol. 196, no. 4, pp. 2319-2331, 2011. 
[17] G. S. Misyris, A. Marinopoulos, D. I. Doukas, T. Tengnér, and D. P. Labridis, "On battery state estimation algorithms for electric ship applications," Electr. Pow. Syst. Res., vol. 151, pp. 115-124, 2017.

[18] H. Rahimi-Eichi, F. Baronti, and M. Y. Chow, "Online adaptive parameter identification and state-of-charge coestimation for Lithium-polymer battery cells," IEEE Trans. Ind. Electron., vol. 61, no. 4, Apr. 2014.

[19] T. Kim, Y. Wang, H. Fang, Z. Sahinoglu, T. Wada, S. Hara, and W. Qiao, "Model-based condition monitoring for lithium-ion batteries," J. Power Sources, vol. 295, pp. $16-27,2015$.

[20] H. Rahimi-Eichi, F. Baronti, and M. Y. Chow, "Modeling and online parameter identification of Li-polymer battery cells for SOC estimation," in Industrial Electronics (ISIE), 2012 IEEE Int. Symposium on, May 2012, pp. 1336-1341.

[21] M. Chen and G. A. Rincon-Mora, "Accurate electrical battery model capable of predicting runtime and I-V performance," IEEE Trans. Energy Convers., vol. 21, no. 2, pp. 504-511, Jun. 2006.

[22] A. Vahidi, A. Stefanopoulou, and H. Peng, "Recursive least squares with forgetting for online estimation of vehicle mass and road grade: theory and experiments," Vehicle Syst. Dyn., vol. 43, no. 1, pp. 31-55, 2005.

[23] N. A. Michailidis, N. A. Bezas, D. I. Doukas, G. S. Misyris, A. G Marinopoulos, and D. P. Labridis, "Battery energy storage systems modeling for online applications comparative analysis of estimation algorithms," in 2017 IEEE PES Innovative Smart Grid Technologies Conference Europe (ISGT-Europe), Turin, Italy, Sep. 2017.

[24] D. Liu, Y. Luo, L. Guo, and Y. Peng, "Uncertainty quantification of fusion prognostics for lithium-ion battery remaining useful life estimation," in 2013 IEEE Conf. on Prognostics and Health Management (PHM), Jun. 2013, pp. 1-8.

[25] G. L. Plett, "Extended Kalman filtering for battery management systems of LiPB-based HEV battery packs: Part 3. state and parameter estimation," J. Power Sources, vol. 134, no. 2, pp. 277-292, 2004.

[26] _ "Sigma-point Kalman filtering for battery management systems of LiPB-based HEV battery packs: Part 2: Simultaneous state and parameter estimation," J. Power Sources, vol. 161, no. 2, p. 1369, 2006.

[27] X. Chen, W. Shen, M. Dai, Z. Cao, J. Jin, and A. Kapoor, "Robust adaptive sliding-mode observer using RBF neural network for Lithiumion battery state of charge estimation in electric vehicles," IEEE Trans. Veh. Technol., vol. 65, no. 4, pp. 1936-1947, Apr. 2016.

[28] T. Kim, Y. Wang, Z. Sahinoglu, T. Wada, S. Hara, and W. Qiao, "State of charge estimation based on a realtime battery model and iterative smooth variable structure filter," in 2014 IEEE Innovative Smart Grid Technologies - Asia (ISGT ASIA), May 2014, pp. 132-137.

[29] M. Petzl and M. A. Danzer, "Advancements in OCV measurement and analysis for Lithium-ion batteries," IEEE Trans. Energy Convers., vol. 28, no. 3, pp. 675-681, Sep. 2013

[30] H. M. Y. Zou, X. Hu and S. E. Li, "Combined state of charge and state of health estimation over lithium-ion battery cell cycle lifespan for electric vehicles," J. Power Sources, vol. 273, pp. 793 - 803, 2015

[31] J. L. S. Lee, J. Kim and B. H. Cho, "State-of-charge and capacity estimation of lithium-ion battery using a new open-circuit voltage versus state-of-charge," J. Power Sources, vol. 185, no. 2, pp. 1367 - 1373, 2008.

[32] B. D. Y. C. Hu and J. Chung, "A multiscale framework with extended Kalman filter for lithium-ion battery SOC and capacity estimation," Appl. Energy, vol. 92, pp. 694 - 704, 2012.

[33] C. C. C. W. X. Shen, K. T. Chau and E. W. C. Lo, "Neural networkbased residual capacity indicator for nickel-metal hydride batteries in electric vehicles," IEEE Trans. Veh. Technol., vol. 54, no. 5, pp. 17051712, Sep. 2005.

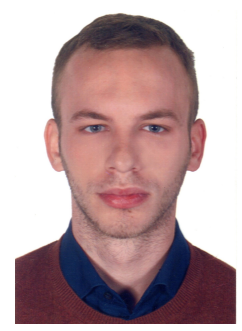

George Misyris (S'15) was born in 1992 in Thessaloniki, Greece. He received the Dipl.-Eng. degree in Electrical and Computer Engineering from the Aristotle University of Thessaloniki, Thessaloniki, Greece. In 2016, he joined ABB Corporate Research, Västerås, Sweden. Since, 2017, he has been working towards the Ph.D. degree at the Denmark Technical University.

His research interests are power systems analysis, operation and control as well as energy storage.

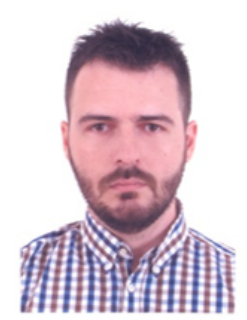

Dimitrios I. Doukas (S'13-M'17) received the Dipl. Eng. and $\mathrm{Ph} . \mathrm{D}$. degrees in electrical and computer engineering from the Aristotle University of Thessaloniki, Greece, in 2009 and 2017, respectively. $\mathrm{He}$ also received the M.Sc. degree in sustainable energy systems from the University of Edinburgh, Edinburgh, UK, in 2011. From 2011 to 2013, he was with ABB Corporate Research, Västerås, Sweden and since 2017 he is with NET2GRID B.V., Zeist, Netherlands.

His research interests are applied superconductivity, energy storage and power system analysis and control.

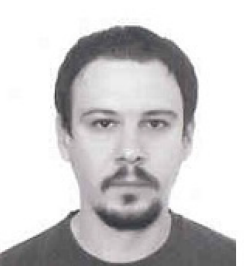

Theofilos A. Papadopoulos (S'01-M'09-SM'18) received the Dipl. Eng. and Ph.D. degrees from the School of Electrical and Computer Engineering at the Aristotle University of Thessaloniki, Greece, in 2003 and 2008, respectively. He is currently Asst. Professor at the Power Systems Laboratory of the Department of Electrical and Computer Engineering of the Democritus University of Thrace, Greece.

His special interests are power systems modeling, PLC and computation of electromagnetic transients. PowerTech '07 Conference. He received the Basil Papadias Award at the IEEE

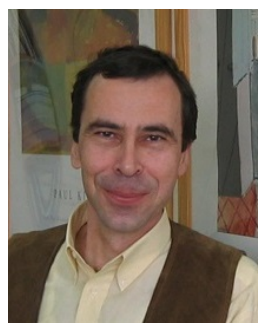

Dimitris P. Labridis (S'88-M'90-SM'00) received the Dipl.-Eng. and Ph.D. degrees from the Department of Electrical Engineering at the Aristotle University of Thessaloniki, Greece, in 1981 and 1989, respectively. Since 1986 he has been with the Electrical Engineering Department, Aristotle University of Thessaloniki, where he is currently a Professor.

His research interests are power system analysis with a special emphasis on the simulation of transmission and distribution systems, smart grids, distributed energy resources, power line communications, coupled electromagnetic and thermal field analysis, interference of power transmission and distribution lines, artificial intelligence applications in power systems and applied superconductivity.

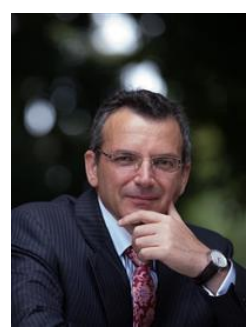

Vassilios G. Agelidis (S'89-M'91-SM'00-F'16) was born in Serres, Greece. He received the B.Eng. degree in electrical engineering from the Democritus University of Thrace, Thrace, Greece, in 1988, the M.S. degree in applied science from Concordia University, Montreal, Canada, in 1992, and the Ph.D. degree in electrical engineering from Curtin University, Australia, in 1997.

He has worked with Curtin University (19931999), University of Glasgow, U.K. (2000-2004), Murdoch University, Perth (2005-2006), the University of Sydney, Sydney, Australia (2007-2010), and the University of New South Wales, Sydney (2010-2016). He is currently a Professor with the Department of Electrical Engineering, Technical University of Denmark, Denmark.

Dr. Agelidis received the Advanced Research Fellowship from the U.K.'s Engineering and Physical Sciences Research Council in 2004. He was the Vice President Operations within the IEEE Power Electronics Society from 2006 to 2007. He was an AdCom Member of the IEEE Power Electronics Society from 2007 to 2009 and the Technical Chair of the 39th IEEE Power Electronics Specialists Conference, Rhodes, Greece, 2008. 